\title{
Second-Order Terahertz Bandpass Frequency Selective Surface With Miniaturized Elements
}

\author{
Amir Ebrahimi, Member, IEEE, Shruti Nirantar, Student Member, IEEE, \\ Withawat Withayachumnankul, Member, IEEE, Madhu Bhaskaran, Member, IEEE, \\ Sharath Sriram, Member, IEEE, Said F. Al-Sarawi, Member, IEEE, and Derek Abbott, Fellow, IEEE
}

\begin{abstract}
In this paper, a second-order frequency selective surface (FSS) made of miniaturized elements is proposed and designed for terahertz applications. The FSS is composed of two layers of metallic arrays separated from each other by a polymer dielectric spacer. The unit cells on the front and back layers are smaller than $\lambda_{0} / 5$, where $\lambda_{0}$ is the free space wavelength. The operation principle of the proposed FSS is described through a circuit model, and a synthesis procedure is presented for designing a desired filtering response. A prototype of the FSS is synthesized to operate at a center frequency of $0.42 \mathrm{THz}$ with $45 \%$ fractional bandwidth. The designed FSS is fabricated by using microfabrication process. The performance is evaluated by using terahertz time-domain spectroscopy. Measurement results show a low sensitivity of the FSS response to oblique angles of incidence for both of the TE and TM polarizations.
\end{abstract}

Index Terms-Bandpass filter, frequency selective surface (FSS), periodic structures, spatial filters, terahertz (THz), THz filter.

\section{INTRODUCTION}

$\mathbf{R}$ APID development of terahertz applications in recent years has been underpinned by numerous designs of passive and active devices and circuits operating over millimeter-wave and terahertz frequencies [1]-[5]. Potential applications of the terahertz radiation include remote sensing [6], imaging [7], chemical and biological sensing [8], [9], etc. Terahertz frequency selective surfaces (THz-FSSs) are key components in many of these applications [10], [11]. A range of FSSs have been reported for remote sensing [12], absorbers or polarization convertors in terahertz systems [13]-[15], sensing and imaging applications [16].

Several FSS structures have been proposed for bandpass filtering at terahertz and millimeter-wave frequencies [17]-[21]. A

Manuscript received January 14, 2015; revised June 04, 2015; accepted June 27, 2015. Date of publication July 24, 2015; date of current version August 31, 2015. (Corresponding Author Amir Ebrahimi.)

A. Ebrahimi, S. F. Al-Sarawi, and D. Abbott are with the School of Electrical \& Electronic Engineering, The University of Adelaide, Adelaide, SA 5005, Australia (e-mail: amir.ebrahimi@adelaide.edu.au).

W. Withayachumnankul is with the School of Electrical \& Electronic Engineering, The University of Adelaide, Adelaide, SA 5005, Australia (e-mail: amir.ebrahimi@adelaide.edu.au). and with the Functional Materials and Microsystems Research Group, School of Electrical \& Electronic Engineering, The University of Adelaide, Adelaide, SA 5005, Australia, and also with the Interdisciplinary Graduate School of Science and Engineering, Tokyo Institute of Technology, Ookayama, Meguro-ku, Tokyo 152-8552, Japan (e-mail: Withawat.withayachumnankul@adelaide.edu.au).

S. Nirantar, M. Bhaskaran, and S. Sriram are with the Functional Materials and Microsystems Research Group, School of Electrical \& Computer Engineering, RMIT University, Melbourne, VIC 3001, Australia (e-mail: sharath. sriram@gmail.com).

Color versions of one or more of the figures in this paper are available online at http://ieeexplore.ieee.org.

Digital Object Identifier 10.1109/TTHZ.2015.2452813 common specification among these conventional FSS structures is that their unit cell dimensions are comparable to half of the operational wavelength. As a result, their frequency response is sensitive to the angle of incidence [22]. In order to circumvent this problem, a new class of FSS structures called miniaturizedelement FSSs (MEFSSs) has been introduced in [23]. These structures are composed of multilayer arrays of non-resonant metallic elements with dimensions much smaller than the operation wavelength. The sub-wavelength unit cells provide a more consistent response of the whole structure with respect to nonplanar phase fronts. Since then, a large number of MEFSS structures for higher-order spatial filtering have been designed and proposed at microwave frequencies [24]-[33]. However, they have not been demonstrated for sub-millimeter-wave and terahertz applications yet. Recently, a MEFSS has been proposed by Moallem and Sarabandi for suppressing an image component provided by a harmonic mixer in MMW radars [34]. The structure in [34] is composed of metallic patches and I-shaped wires that provide a first-order bandpass response with an adjacent transmission zero. Higher-order filters with wide out-ofband rejection are required in certain applications to enhance the spectral selectivity [17]-[21]. Particularly, FSSs with these specifications can reduce the radar cross section (RCS) of antennas, and reduce undesirable strong background noise in spectroscopy [35]. Based on this requirement, this article introduces a MEFSS with a second-order filter response for sub-millimeterwave and terahertz applications with a wide rejection band and large angular tolerance. The proposed structure is composed of two layers of metallic arrays each having a first-order filter response. Since the structure is made of miniaturized unit cells, it's electromagnetic response can be modelled through a lumped element equivalent circuit [36]-[38]. Therefore, an analytical procedure can be developed for the proposed FSS structure by using the standard filter theory leading to a straightforward design. This article is organized as follows. The design procedure and the operation principle of the proposed FSS is presented in Section II. Section III explains the fabrication process of the designed FSS. Experimental results for the fabricated sample are presented and discussed in Section IV and finally, Section V provides the conclusion.

\section{FSS Topology AND Design Procedure}

\section{A. FSS Structure and Equivalent Circuit Model}

A three-dimensional view of the proposed terahertz FSS is shown in Fig. 1(a). The structure is composed of two periodic metallic arrays that are separated from each other by a dielectric 


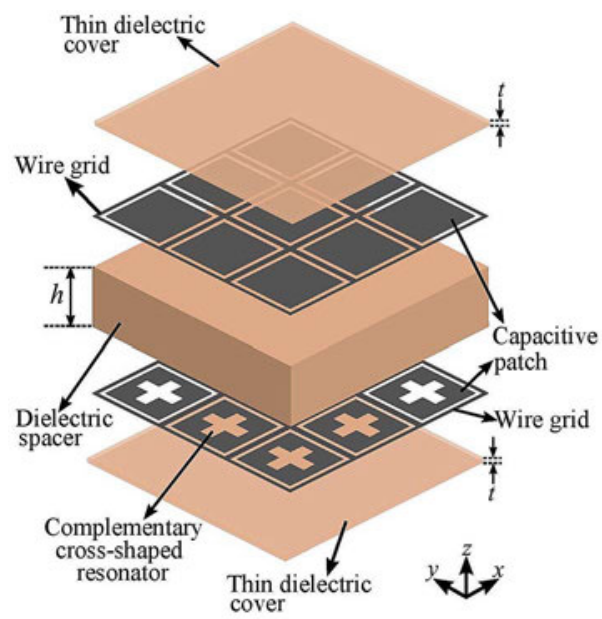

(a)
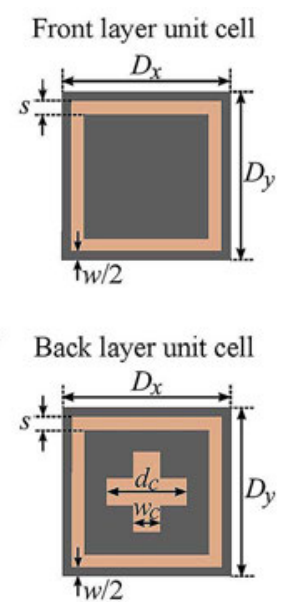

(b)
Fig. 1. Topology of the proposed second-order terahertz FSS. (a) 3-D view of the FSS with the gray regions showing the metallization and the light brown regions showing the dielectric spacer. (b) Unit cells on the front and back layers along with their geometric parameters.

spacer having a subwavelength thickness of $h$. Fig. 1(b) shows the unit cells for the front and back layers. The dimensions of each unit cell are $D_{x}$ and $D_{y}\left(D_{x}=D_{y}=D\right)$ along the $x$ and $y$ directions, respectively. The front layer unit cell is made of a wire loop surrounding a non-resonant capacitive square patch. The back layer unit cell is very similar to the front layer excepting a complementary cross-shaped resonator (CCSR) that is embedded into the capacitive patch. Both of the front and back 2-D metallic arrays are covered by a very thin protective layer (thickness $=t$ ) made of the same dielectric material as the spacer.

As mentioned before, since the structure is composed of miniaturized unit cells, it can be modeled by a lumped-element equivalent circuit. The equivalent circuit model of this FSS is depicted in Fig. 2(a), which is valid for a normally incident plane wave. In this model, $L_{1}$ and $L_{2}$ account for the inductive behavior of the front and back layers wire grids. The capacitors $C_{L 1}$ and $C_{L 2}$ model the capacitive effect between the square patch and the inductive wire grid in the front and back layers, respectively. The CCSR in the back layer is modelled by a $L_{3} C_{3}$ resonator. The dielectric spacer that separates the two layers is modelled through a section of transmission line with a characteristic impedance of $Z_{T}$ and length of $h$. It should be mentioned that $Z_{T}=Z_{0} / \sqrt{\epsilon_{r}}$, where $Z_{0}=377 \Omega$ is the free space impedance and $\epsilon_{r}$ is the relative permittivity of the spacer. And finally, the Ohmic loss associated with the front and back wire grids and CCSR are modeled by resistors $R_{1}, R_{2}$ and $R_{3}$ respectively. These losses are related to the surface resistivity of the metallization and the current distribution therein [34]. The combination of the complementary cross-shaped resonator $\left(L_{3} C_{3}\right)$ and $C_{L 2}$ adds a transmission zero to the filter response and improves it's out of band behavior. In fact, this transmission zero appears at the frequency when the combination of $C_{L 2}$ and $L_{3} C_{3}$ is short-circuited. Thus, this frequency is given by

$$
f_{\mathrm{z}}=\frac{1}{2 \pi \sqrt{L_{3}\left(C_{3}+C_{L 2}\right)}} .
$$

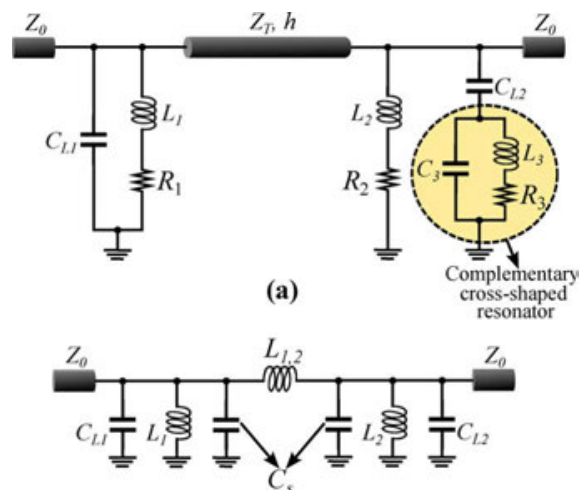

(b)

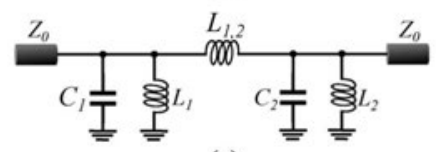

(c)

Fig. 2. Equivalent circuit model of the proposed FSS for the normaly incident EM wave. (a) A full circuit model for normal incidence. (b) A simplified model with the transmission line section replaced with equivalent lump elements and the loss $R$ neglected. (c) Standard circuit model of a second-order bandpass coupled resonator filter. This circuit is equivalent to the circuit in Fig. 2(b) with $C_{1}=C_{L 1}+C_{\mathrm{s}}$ and $C_{2}=C_{L 2}+C_{\mathrm{s}}$.

This transmission zero can be tuned by varying the dimensions of the complementary cross-shaped resonator $\left(d_{c}, w_{c}\right)$, which in turn translates to the variation of $L_{3}$ and $C_{3}$ values.

Since the values of $L_{3}$ and $C_{3}$ are designed to be much smaller than $L_{2}$ and $C_{L 2}$, the parallel combination of $L_{3} C_{3}$ has a very small impedance around the passband frequencies of the FSS. So, $L_{3} C_{3}$ can be considered as a short circuit at these frequencies. In addition, the transmission line section can be replaced with it's equivalent lumped-element circuit model by using the telegrapher's equation [39]. Fig. 2(b) illustrates the FSS equivalent circuit around the passband by considering $L_{3} C_{3}$ as a short circuit and replacing the transmission line with its circuit model $\left(L_{1,2}\right.$ and $\left.C_{\mathrm{s}}\right)$. The losses are not considered in Fig. 2(b) for simplicity. The values of $L_{1,2}$ inductor and $C_{\mathrm{s}}$ capacitors can be obtained by using the following relations [22]

$$
\begin{aligned}
L_{1,2} & =\mu_{0} \mu_{r} h, \\
C_{\mathrm{s}} & =\frac{\epsilon_{0} \epsilon_{r} h}{2}
\end{aligned}
$$

where $\mu_{0}$ and $\mu_{r}$ are the permeability of the free space and the relative permeability of the spacer, respectively. Likewise, $\epsilon_{0}$ and $\epsilon_{r}$ are the permittivity of the free space and the relative permittivity of the spacer, respectively. The circuit of Fig. 2(b) can be converted into the one in Fig. 2(c) that shows a standard basic circuit model of a second-order bandpass filter [40]. In this form, $C_{1}=C_{L 1}+C_{\mathrm{s}}$ and $C_{2}=C_{L 2}+C_{\mathrm{s}}$, and the circuit can be considered as coupled input and output resonators of $L_{1} C_{1}$ and $L_{2} C_{2}$, respectively.

\section{B. Synthesis Procedure of the Proposed FSS}

As discussed above, the frequency response of the considered FSS around its passband can be described by the circuit model in Fig. 2(c). By using this circuit model, a desired second-order 
filtering response can be synthesized and then, the equivalent circuit parameters can be converted into the corresponding geometrical parameters of the proposed FSS. The lumped-element values of the equivalent circuit in Fig. 2(c) can be determined by applying the concept of coupled-resonator filter with inductive coupling [36], [40]. Supposing that $f_{0}$ is the center frequency of the filter and $\delta=\mathrm{BW} / f_{0}$ is the fractional bandwidth, the values of the circuit elements in Fig. 2(c) can be obtained based on the following steps:

1) The values of the $C_{1}$ and $C_{2}$ capacitors can be determined from the following relation:

$$
C_{i}=\frac{q_{i}}{\omega_{0} r_{i} Z_{0} \delta}, \quad i=1,2 .
$$

In (4), $Z_{0}=377 \Omega, q_{1}$ and $q_{2}$ are the normalized quality factors of the input and output resonators, and $r_{1}$ and $r_{2}$ are the normalized source and load impedances of the filter in Fig. 2(c) [40].

2) The values of $L_{1}$ and $L_{2}$ inductors can be obtained by

$$
L_{i}=\frac{1}{\omega_{0}^{2}\left(C_{i}-k_{1,2} \delta \sqrt{C_{1} C_{2}}\right)}, \quad i=1,2
$$

where $k_{1,2}$ is the normalized coupling coefficient between the input and output resonators.

3) The coupling inductance $L_{1,2}$ can be calculated from

$$
L_{1,2}=\frac{1}{\omega_{0}^{2} k_{1,2} \delta \sqrt{C_{1} C_{2}}} .
$$

The normalized quality factors and coupling coefficient for some different filter types are extracted from [40] and presented in Table I.

After calculating the values of the circuit elements in Fig. 2(c), the thickness of the dielectric spacer that separates the front and back metallic arrays can be obtained from (2). Then the spacer capacitance $C_{\mathrm{s}}$ can be derived from (3), and the values of $C_{L 1}$ and $C_{L 2}$ capacitors can be found from (7).

$$
C_{L i}=C_{i}-\frac{\epsilon_{0} \epsilon_{r} h}{2}, \quad i=1,2 .
$$

In order to convert the calculated lumped element values into the geometrical parameters, the method presented in [36]-[38] can be used. The effective inductance of an infinite wire grid shown in Fig. 3(a) with a period of $l$ and a wire width of $w$ is given by [22]

$$
L=\mu_{0} \mu_{\mathrm{eff}} \frac{l}{2 \pi} \ln \left(\frac{1}{\sin \frac{\pi w}{2 l}}\right)
$$

where $\mu_{0}$ is the permeability of the free space and $\mu_{\text {eff }}$ is the effective permeability of the substrate. Equation (8) can be used for calculating the wires width in the front and back layer metallization of the proposed FSS. The period of the wire grid is considered to be equal to the unit cell size in Fig. $1(l=D)$ and the unit cell size can be chosen arbitrarily. However, choosing very small unit cell size leads to a very thin wire that might not be practical due to fabrication limits. It is worth mentioning that (8) is valid when the wire grid is away from any metallic objects such as capacitive patches in Fig. 2 or scatterers [36], [38]. So, the value of $w$ calculated from (8) can only be used as an approximation. A more accurate value of $w$ can be obtained
TABLE I

NORMALIZED QUALITY FACTOR AND COUPLING COEFFICIENTS FOR DIFFERENT FILTER TYPES EXTRACTED FROM [40]

\begin{tabular}{cccccc}
\hline \hline Filter Type & $q_{1}$ & $q_{2}$ & $k_{12}$ & $r_{1}$ & $r_{2}$ \\
\hline Gaussian & 0.4738 & 2.185 & 0.9828 & 1 & 1 \\
Butterworth & 1.4142 & 1.4142 & 0.70711 & 1 & 1 \\
Chebyshev (0.01 dB ripple) & 1.4829 & 1.4829 & 0.7075 & 1 & 1.1007 \\
Chebyshev (0.1 dB ripple) & 1.6382 & 1.6382 & 0.7106 & 1 & 1.3554 \\
Chebyshev (0.5 dB ripple) & 1.9497 & 1.9497 & 0.7225 & 1 & 1.9841 \\
\hline \hline
\end{tabular}

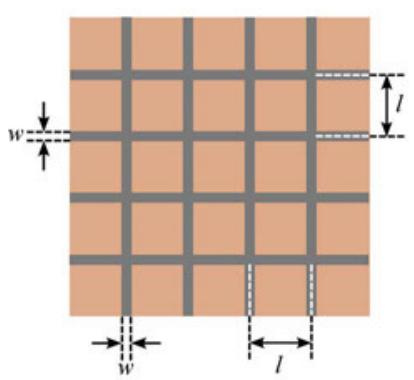

(a)

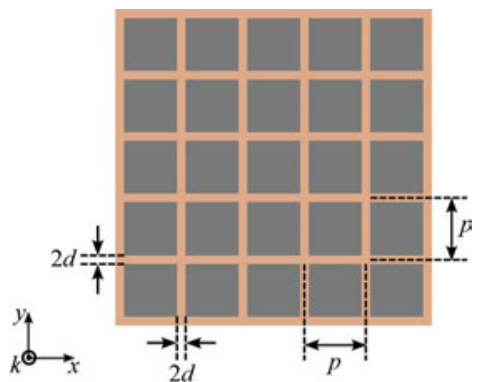

(b)
Fig. 3. (a) Infinite metallic wire grid. (b) Infinite metallic square patch array. The wire grid shows an inductive behavior whereas the patch array shows a capacitive effect with respect to the incident electromagnetic wave. Metallization is indicated with gray colored regions.

by fitting the full-wave simulation result to the analytical circuit calculation.

The effective capacitance of an infinite array of sub-wavelength capacitive square patches shown in Fig. 3(b) can be obtained by using [22]

$$
C=\epsilon_{0} \epsilon_{\mathrm{eff}} \frac{2 p}{\pi} \ln \left(\frac{1}{\sin \frac{\pi d}{2 p}}\right) .
$$

In (9), $\epsilon_{0}$ is the permittivity of free space and $\epsilon_{\mathrm{eff}}$ is the effective permittivity of the dielectric spacer, $p$ is the period of the array and $2 d$ is the gap spacing between the adjacent patches. Although for the proposed structure, the capacitive patches are surrounded by the wire grid, (9) can still be used as an approximation if the capacitive effect between the edges of the metallic square patches and the wire grid is approximated by a corresponding patch array in Fig. 3(b). To this end, the gap spacing between the adjacent metallic patches in Fig. 3(b) is assumed to be equal to the space between a capacitive patch and the wire grid in Fig. 1(b) $(2 d=s)$. By this assumption, the period of the corresponding patch array of Fig. 3(b) will be

$$
p=D-w-s,
$$

where, $D=D_{x}=D_{y}$ is the unit cell size in Fig. 2(b) and $w$ is the width of the wire grid surrounding each capacitive patch. Based on (9) and (10), the spacing between the patches in Fig. 3(b), that corresponds to the spacing between the patches and the wire grid in Fig. 2 can be determined.

\section{Design of Terahertz FSS}

Based on the synthesis procedure in Section II-B, this section demonstrates a design of a second-order bandpass FSS at terahertz frequencies. The design starts by considering a center fre- 


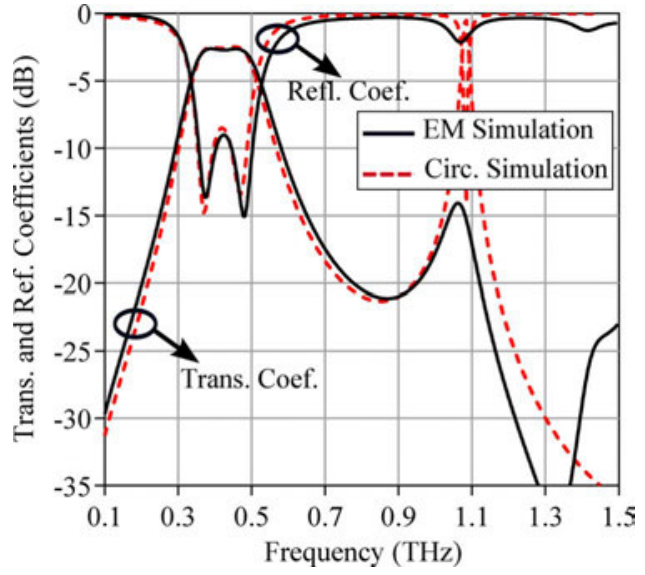

Fig. 4. Simulation results of the proposed FSS for the normal incident angle. The parameters used in simulations are: $C_{L 1}=C_{L 2}=4.2 \mathrm{fF}, L_{1}=L_{2}=$ $45 \mathrm{pH}, R_{1}=R_{2}=6.5 \Omega, D_{x}=D_{y}=130 \mu \mathrm{m}, h=100 \mu \mathrm{m}, t=10 \mu \mathrm{m}$, $w=10 \mu \mathrm{m}$ and $s=5 \mu \mathrm{m}$.

quency of $f_{0}=0.42 \mathrm{THz}$ and $\mathrm{a}-3 \mathrm{~dB}$ fractional bandwidth of $\delta=35 \%$ for the circuit model in Fig. 2(c). This frequency band is chosen to match the capabilities of most terahertz time-domain spectroscopy (THz-TDS) systems. Polydimethylsiloxane (PDMS) is considered for the dielectric spacer since it has a low dielectric constant of $\epsilon_{r}=2.35$ causing a low impedance mismatch with free space [34]. The front and back metallic layers are made of $200 \mathrm{~nm}$ thick gold $(\mathrm{Au})$ with the DC conductivity of $4.1 \times 10^{7} \mathrm{~S} / \mathrm{m}$ and the sheet resistance of around $0.2+j 0.22 \Omega$ /square at the passband of the FSS. In order to improve the mechanical strength of the filter, two encapsulating PDMS layers with a thickness of $10 \mu \mathrm{m}$ are applied to the front and back metal layers. The relative permeability of the PDMS is $\mu_{r}=1$. By assuming a Butterworth filter response, the values of the circuit elements in Fig. 2(a) are calculated using the synthesis method described in Section II-B. The calculated values are $C_{1}=C_{2}=4.23 \mathrm{fF}, L_{1}=L_{2}=45 \mathrm{pH}$ and $L_{1,2}=$ $130 \mathrm{pH}$. The unit cell size is considered as $D_{x}=D_{y}=D$ $=130 \mu \mathrm{m}$, which is slightly smaller than the $\lambda_{0} / 5$, where $\lambda_{0}$ is the free space wavelength at $0.42 \mathrm{THz}$. By using the procedure described in Section II-B, the circuit parameters can be mapped into the geometrical dimensions of the FSS: $w=14.8 \mu \mathrm{m}$, $s=4.5 \mu \mathrm{m}$ and $h=103 \mu \mathrm{m}$. Now, $w$ and $s$ can be optimized in simulation for obtaining more accurate values. Equation (9) is reasonably accurate since the capacitance between the patches in Fig. 3(b) corresponds directly to the capacitance between the patches and grid in Fig. 1. On the other hand, the presence of those square metallic patches in the vicinity of the wire grid affects the magnetic field and hence reduces the inductance. So, the final optimized value of $w$ is expected to be smaller than the value obtained from (8). For optimization, we first consider the calculated value of $w$ from (8) based on the synthesized value of $L_{1}$ and $L_{2}$. Now, by having $D$ and $w$, the value of $s$ will be determined from (9) to satisfy the synthesized value of $C_{1}$ and $C_{2}$. The unit cell is then simulated to obtain the $L$ and $C$ values. If the inductance of the wire grid obtained from the simulation is smaller than the synthesized value, the previous steps are then repeated with a smaller value for $w$, until

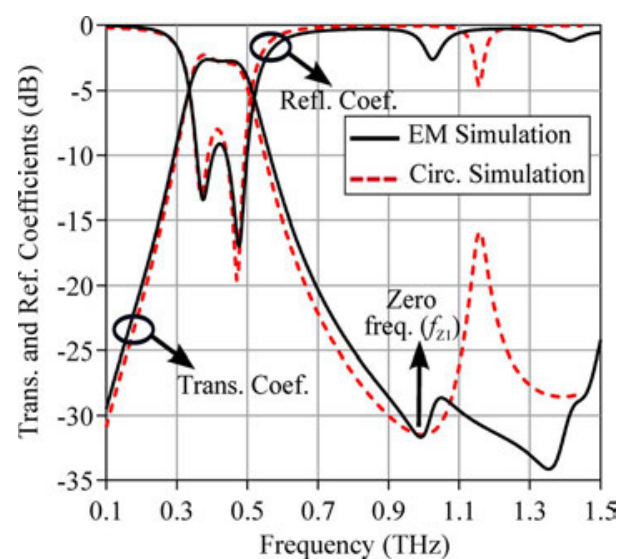

Fig. 5. Simulation results of the proposed FSS for the normal incident angle by considering the effect of the complementary cross-shaped resonator. The complementary cross shaped resonator dimensions are: $w_{c}=20 \mu \mathrm{m}, d_{c}=65 \mu \mathrm{m}$ and $L_{3}=3 \mathrm{pH}, C_{3}=2.9 \mathrm{fF}, R_{2}=1.2 \Omega$ and $R_{3}=4.25 \Omega$ are considered in circuit model simulations. The rest of parameters are the same as the Fig. 4.

desired consistency between the simulation results and the synthesized values is achieved. The optimized equivalent circuit and geometrical parameters of the designed FSS are listed in the caption of Fig. 4. As seen, the optimized value for $s$ is very close to the value obtained from (9) but, the optimized value of $w$ is smaller than the value predicted by (8).

After mapping the synthesized values of the $L$ and $C$ parameters to the geometrical parameters of the FSS, the values of the resistances in Fig. 2 can be determined by curve fitting of the EM and circuit simulation results. Fig. 4 shows a comparison between the results obtained from the circuit simulation with ADS and from the full-wave electromagnetic simulation with CST Microwave Studio. Both of the transmission and reflection coefficients are shown. As can be observed in Fig. 4, there is a good agreement between the circuit model and the full-wave electromagnetic simulation of the FSS. The good agreement confirms that the circuit based synthesis procedure offers an efficient analytical method for designing the proposed FSS configuration.

The results in Fig. 4 are obtained without considering the effect of the CCSR. As explained in Section II-A, the combination of CCSR $\left(L_{3}, C_{3}\right)$ and $C_{L 2}$ adds a transmission zero that can be used to improve the out of band response of the FSS. The frequency of this zero can be calculated from (1) and can be tuned by varying $w_{c}$ and $d_{c}$. Fig. 5 shows the transmission response of the FSS by considering $w_{c}=20 \mu \mathrm{m}$ and $d_{c}=65 \mu \mathrm{m}$. As seen, the out of band response in Fig. 5 is improved with respect to Fig. 4 since the transmission coefficient stays below $-27 \mathrm{~dB}$ up to $1.45 \mathrm{THz}$.

The values of $L_{3}$ and $C_{3}$ elements that represent the effect of the CCSR can be determined by a semi-analytical procedure described as follows: the zero frequency provided by $L_{3}$, $C_{3}$ and $C_{L 2}$ can be calculated from (1). The value of $C_{L 2}$ as listed in caption of Fig. 5 is already designed by the synthesis procedure. On the other hand, the zero frequency is obtained from EM simulation in CST Microwave Studio ( $f_{\mathrm{Z} 1}$ in Fig. 5). By putting arbitrary capacitors of $C_{a r}$ between the capacitive square patches and the wire grid in the back layer of the FSS in CST Microwave Studio, the capacitance between the wire grid 
and square patches will be increased to $C_{L 2}+C_{a r}$. This will shift down the zero frequency to

$$
f_{\mathrm{Z} 2}=\frac{1}{2 \pi \sqrt{L_{3}\left(C_{3}+C_{L 2}+C_{a r}\right)}} .
$$

So, by solving (1) and (11), the unknown values of $L_{3}$ and $C_{3}$ are determined. The calculated $L_{3}$ and $C_{3}$ for the designed FSS are given in Fig. 5. It should be noted that the circuit model in Fig. 2(a) and the results in Fig. 5 are only valid for normal incidence. For oblique incidence, both of the free-space equivalent impedance $Z_{0}$ and the dielectric spacer impedance $Z_{T}$ need to be changed to

$$
\begin{aligned}
Z_{0, \mathrm{TE}} & =\frac{Z_{0}}{\cos \theta} \\
Z_{0, \mathrm{TM}} & =Z_{0} \cos \theta \\
Z_{T, \mathrm{TE}} & =\frac{Z_{0, \mathrm{TE}}}{\sqrt{\epsilon_{r}}} \\
Z_{T, \mathrm{TM}} & =\frac{Z_{0, \mathrm{TM}}}{\sqrt{\epsilon_{r}}}
\end{aligned}
$$

where $Z_{0}=377 \Omega$ is the free-space characteristic impedance. In addition, the front and back layers equivalent impedance need to be modified for the oblique incidence [41]. A model describing the effect of oblique incidence on the impedance of the layers and the couplings in higher order Floquet modes is available for the square patch array [41], [42]. This model gives an insightful understanding of the FSS response under oblique incidence and in higher order Floquet modes.

\section{FABRicAtion PROCESS}

The FSS structure is fabricated to experimentally validate the simulation results by utilizing standard microfabrication techniques. The FSS is composed of alternating three layers of PDMS and two layers of patterned metal. In the first step, as a supporting substrate, a 3 inch silicon wafer is coated with $20 \mathrm{~nm}$ aluminium (Al), which enables final release of the multi-layer structure from silicon in a freestanding form. A $10 \mu \mathrm{m}$ PDMS layer acting as encapsulation is spin-coated on the Al-coated silicon wafer. First metallization step is performed with $200 \mathrm{~nm}$ gold $(\mathrm{Au})$ with a $20 \mathrm{~nm}$ adhesion layer of chromium $(\mathrm{Cr})$ deposited by electron beam evaporation (PVD75, Kurt J. Lesker) at a rate of $0.1 \mathrm{~nm} / \mathrm{s}$. Metal layers are pattered by photolithography followed by wet chemical etching [2], [3]. Photoresist is then stripped off with acetone, rinsed with isopropanol and dried with high purity nitrogen.

Then, a second PDMS layer of $100 \mu \mathrm{m}$ thickness, acting as dielectric spacer, is spun-on and cured on a leveled surface at room temperature to ensure thickness uniformity. Subsequently, the second layer of metallization followed by photolithography with alignment and etching is performed. To encapsulate the second level of metallization, a third PDMS layer of $10 \mu \mathrm{m}$ is spun-on to complete the fabrication. The resulting structure is then peeled off from the supporting silicon and transferred onto a 3 inch clamp for testing. Fig. 6 shows micrographs of the front and back metal layers and the fabricated structure supported in a clamp for testing.

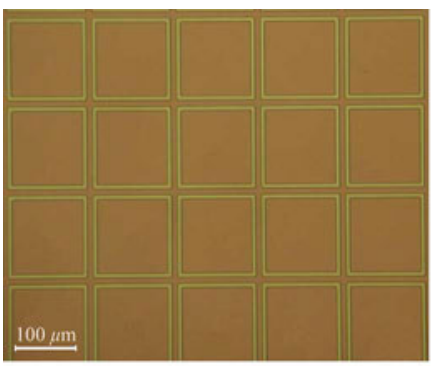

(a)

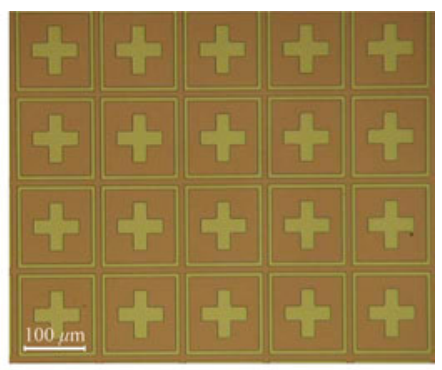

(b)

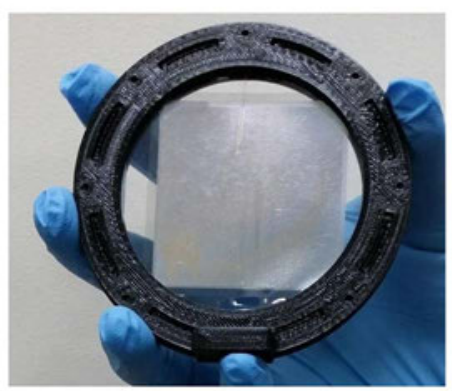

(c)

Fig. 6. Fabricated second-order terahertz FSS. Optical micrographs of the (a) front and (b) back layers. (c) Frame-supported MEFSS after release.

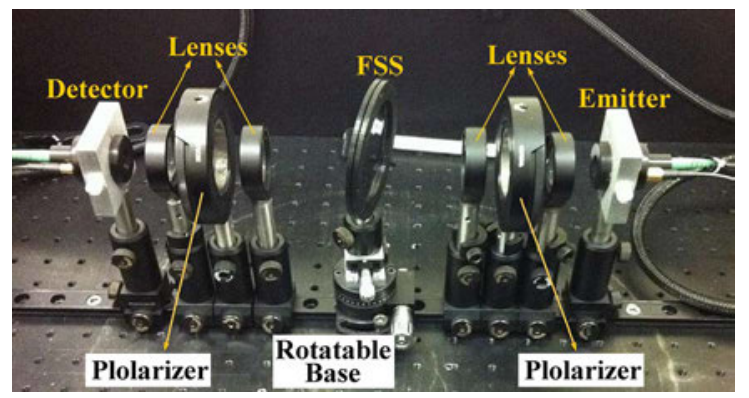

Fig. 7. Free-space measurement setup. A rotatable base is considered for measuring the FSS performance at oblique angles of incidence.

\section{Results AND Discussion}

This section presents the simulation and experimental measurement results to evaluate the performance of the designed FSS under different conditions. The free-space measurement is performed using the Menlo Systems GmbH Tera K15. The measurement setup is shown in Fig. 7. The setup is composed of Tera 15-SL25-FC and Tera 15-DP25-FC antenna modules acting as emitter and detector respectively that provide 2-port S-parameter measurement. The four identical lenses are made of polymer with a diameter of $50 \mathrm{~mm}$, an effective focal length of $54 \mathrm{~mm}$, and a working distance of $46 \mathrm{~mm}$. A rotatable base is used for the FSS to evaluate its response for different oblique angles of incidence. The emitter and detector can be rotated around the propagation axis to obtain TE and TM polarizations. Two polarizers are placed between the lenses to have better polarization purity in TE and TM mode measurements. A broadband terahertz pulse is radiated from the emitter through the FSS to the detector. The four lenses collimate and focus the beam onto the FSS. The transmission response is then normalized with a response obtained from free-space measurement.

In Fig. 8, the measured transmission response of the fabricated FSS is plotted together with the electromagnetic and 


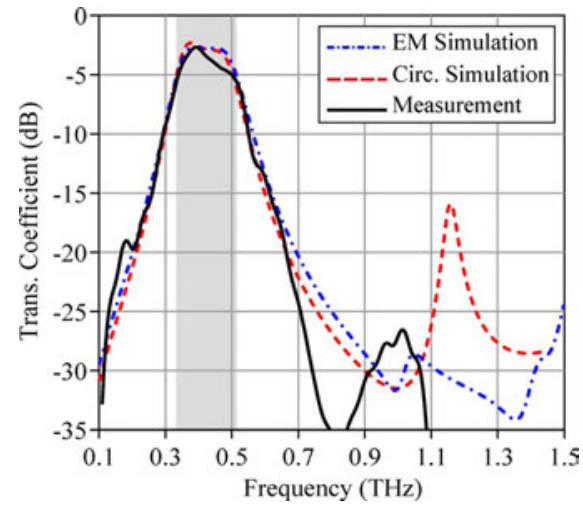

Fig. 8. Comparison between the simulation and measurement results of the FSS transmission coefficient under normal angle of incidence. The $-3 \mathrm{~dB}$ bandwidth is shaded with gray.

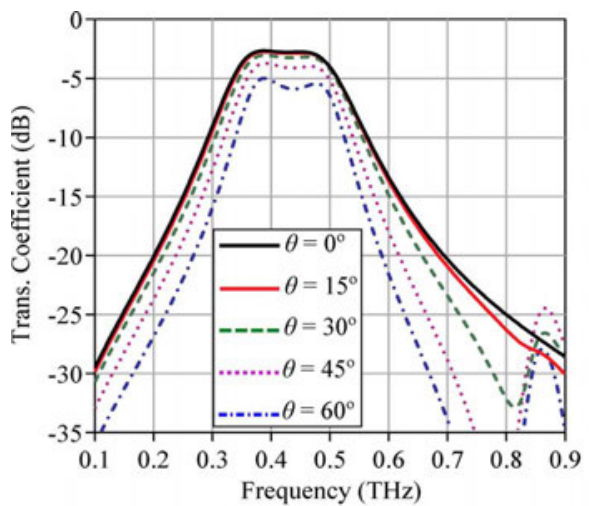

(a)

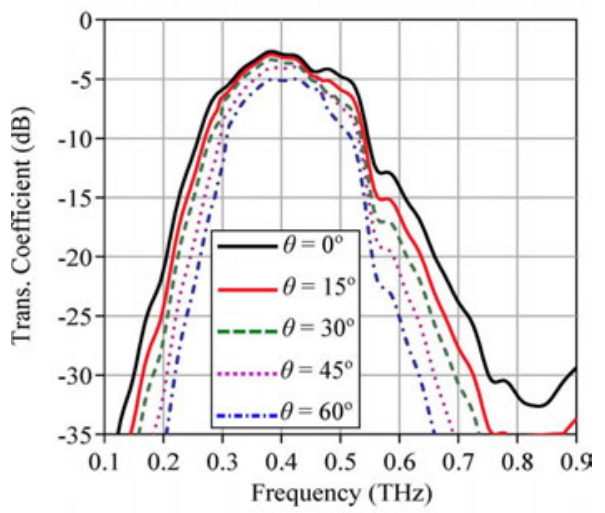

(b)

Fig. 9. Transmission responses of the proposed FSS for oblique angles of incidence with TE polarization. (a) Simulated transmission responses in CST Microwave Studio. (b) Measured transmission responses.

equivalent circuit simulation results at normal angle of incidence. As seen in this figure, a good agreement between the simulation and measurement results is achieved within the measurement bandwidth. The center frequency is $0.42 \mathrm{THz}$ and the $-3 \mathrm{~dB}$ bandwidth of the passband is around $45 \%$. This is wider than the designed $35 \%$ bandwidth because the dissipation losses decrease the quality factor $(Q)$ of the front and back layers and hence increase the bandwidth. A wide out-of-band rejection is obtained up to $1.5 \mathrm{THz}$ below $-25 \mathrm{~dB}$. The maximum loss in the passband is less than $5 \mathrm{~dB}$. This loss is mainly attributed to the dielectric loss in the PDMS layers. Additional simulations (not

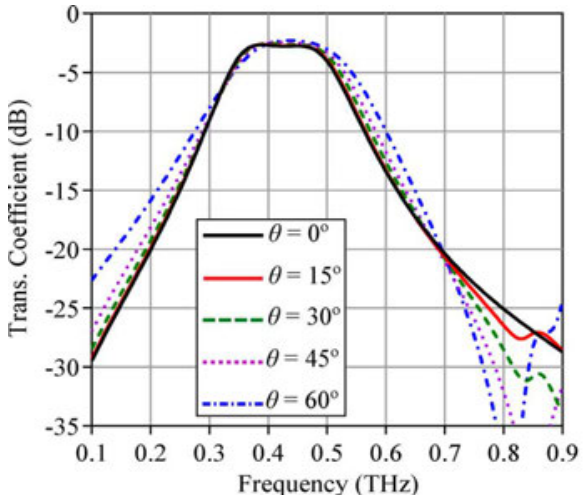

(a)

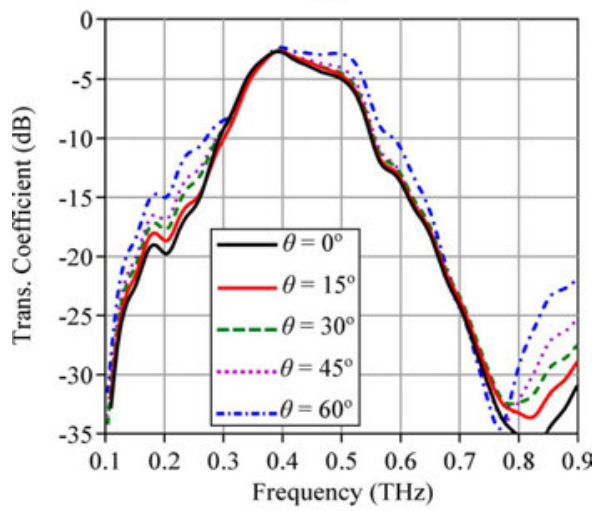

(b)

Fig. 10. Transmission responses of the proposed FSS for oblique angles of incidence with TM polarization. (a) Simulated transmission responses in CST Microwave Studio. (b) Measured transmission responses.

shown) reveal that by replacing PDMS layers with a lower loss material such as cyclo-olefin copolymer (COC), the insertion loss in passband is reduced to $1 \mathrm{~dB}$. The discrepancies between the measured and EM simulation results in the out-of-band response is attributed to the limited dynamic range of the measurement system.

The performance of the proposed FSS for the oblique angles of incidence is also investigated through simulation and measurement. The simulated and measured transmission coefficients of the FSS for the TE polarization are shown in Fig. 9. In addition, Fig. 10 presents the simulated and measured transmission coefficients for the TM polarization. In both cases, the results are presented for $0^{\circ}-60^{\circ}$ incidence angles. As observed, the FSS can reasonably sustain its original response at very large incidence angles. However, the fractional bandwidth (FBW) is decreased for the TE polarization response as the angle of incidence increases. On the contrary, for the TM polarization, the FBW is increased as the incidence angle increases. This is mainly due to a change in the wave impedance for the two modes as described through (12)-(15) [43]. So, as the angle of incidence increases, $Z_{0, \mathrm{TE}}$ will be increased resulting in a larger loaded quality factor in the input/output resonators that causes a smaller fractional bandwidth. On the other hand, in the TM mode, $Z_{0, \mathrm{TM}}$ will be decreased by increasing $\theta$, and thus leads to a smaller loaded quality factor in the input/output resonators and a larger fractional bandwidth. A small discrepancy between the measured TE and TM transmission responses under normal 
incidence is possibly caused by a small difference in the system alignment and by a small anisotropy in the sample.

\section{CONCLUSION}

A second-order bandpass FSS based on miniaturized elements has been proposed and implemented for terahertz applications. An equivalent circuit is considered for modelling the FSS response. A simple synthesis procedure has been developed based on the equivalent circuit and the standard filter theory for designing the proposed FSS. The presented synthesis procedure has been verified through both simulation and experimental results. The results show a wide out-of-band rejection below $-25 \mathrm{~dB}$ up to $1.5 \mathrm{THz}$. In addition, the spectral responses of the FSS are consistent over a wide range of incidence angles for both the TE and TM polarizations. The transmission level of the FSS in the passband can be improved significantly by a low loss polymer such as $\mathrm{COC}$ for dielectric layers.

\section{ACKNOWLEDGMENT}

The authors would like to acknowledge H. Ho of the School of Electrical and Electronic Engineering, The University of Adelaide, Adelaide, Australia, for his technical assistance during the FSS measurement. They also acknowledge A. Upadhyay, P. Thurgood, and P. Gutruf of the Functional Materials and Microsystems Research Group, RMIT University, Melbourne, Australia, for their assistance in fabrication of the FSS prototype.

\section{REFERENCES}

[1] A. Horestani, W. Withayachumnankul, A. Chahadih, A. Ghaddar, M. Zehar, D. Abbott, C. Fumeaux, and T. Akalin, "Metamaterial-inspired bandpass filters for terahertz surface waves on Goubau lines," IEEE Trans. THz Sci. Technol., vol. 3, no. 6, pp. 851-858, Nov. 2013.

[2] T. Niu, W. Withayachumnankul, B. S.-Y. Ung, H. Menekse, M. Bhaskaran, S. Sriram, and C. Fumeaux, "Experimental demonstration of reflectarray antennas at terahertz frequencies," Opt. Express, vol. 21, no. 3, pp. 2875-2889, 2013.

[3] T. Niu, W. Withayachumnankul, A. Upadhyay, P. Gutruf, D. Abbott, M. Bhaskaran, S. Sriram, and C. Fumeaux, "Terahertz reflectarray as a polarizing beam splitter," Opt. Express, vol. 22, no. 13, pp. 16 148-16 $160,2014$.

[4] J. E. Heyes, W. Withayachumnankul, N. K. Grady, D. R. Chowdhury, A. K. Azad, and H.-T. Chen, "Hybrid metasurface for ultra-broadband terahertz modulation," Appl. Phys. Lett., vol. 105, no. 18, 2014.

[5] A. Jam, M. Moallem, J. East, and K. Sarabandi, "A non-contact waveguide probe for on-wafer s -parameter measurements for submillimeter-wave to terahertz band," IEEE Trans. THz Sci. Technol., vol. 4, no. 4, pp. 515-522, Jul. 2014.

[6] R. Martin and D. Martin, "Quasi-optical antennas for radiometric remote-sensing," Electron. Commun. Eng. J., vol. 8, no. 1, pp. 37-48, 1996.

[7] R. Appleby and R. N. Anderton, "Millimeter-wave and submillimeterwave imaging for security and surveillance," Proc. IEEE, vol. 95, no. 8, pp. 1683-1690, 2007.

[8] W. Withayachumnankul, H. Lin, K. Serita, C. M. Shah, S. Sriram, M. Bhaskaran, M. Tonouchi, C. Fumeaux, and D. Abbott, "Sub-diffraction thin-film sensing with planar terahertz metamaterials," Opt. Express, vol. 20, no. 3, pp. 3345-3352, 2012.

[9] P. Siegel, "Terahertz technology in biology and medicine," IEEE Trans. Microw. Theory Techn., vol. 52, no. 10, pp. 2438-2447, Oct. 2004.

[10] A. Ebrahimi, W. Withayachumnankul, S. Al-Sarawi, and D. Abbott, "Second-order bandpass frequency selective surface for terahertz applications," in 2014 39th Int. Conf. on Infrared, Millim. THz Waves (IRMMW-THz), Sep. 2014, pp. 1-2.
[11] S. Vegesna, Y. Zhu, A. Bernussi, and M. Saed, "Terahertz two-layer frequency selective surfaces with improved transmission characteristics," IEEE Trans. THz Sci. Technol., vol. 2, no. 4, pp. 441-448, Jul. 2012.

[12] R. Dickie et al., "Submillimeter wave frequency selective surface with polarization independent spectral responses," IEEE Trans. Antennas Propagat., vol. 57, no. 7, pp. 1985-1994, Jul. 2009.

[13] Y. Z. Cheng, W. Withayachumnankul, A. Upadhyay, D. Headland, Y. Nie, R. Z. Gong, M. Bhaskaran, S. Sriram, and D. Abbott, "Ultrabroadband plasmonic absorber for terahertz waves," Adv. Optical Mater., 2014.

[14] M. Euler, V. Fusco, R. Cahill, and R. Dickie, "325 GHz single layer sub-millimeter wave FSS based split slot ring linear to circular polarization convertor," IEEE Trans. Antennas Propagat., vol. 58, no. 7, pp. 2457-2459, Jul. 2010.

[15] Y. Z. Cheng, W. Withayachumnankul, A. Upadhyay, D. Headland, Y. Nie, R. Z. Gong, M. Bhaskaran, S. Sriram, and D. Abbott, "Ultrabroadband reflective polarization convertor for terahertz waves," Appl. Phys. Lett., vol. 105, no. 18, 2014.

[16] J. J. Sanz Fernandez, "Frequency Selective Surfaces for Terahertz Applications," Ph.D., University of Edinburgh, Edinburgh, U.K., 2012.

[17] Y. Zhu, S. Vegesna, V. Kuryatkov, M. Holtz, M. Saed, and A. A. Bernussi, "Terahertz bandpass filters using double-stacked metamaterial layers," Opt. Lett., vol. 37, no. 3, pp. 296-298, 2012.

[18] F. Lan, Z. Yang, L. Qi, X. Gao, and Z. Shi, "Terahertz dual-resonance bandpass filter using bilayer reformative complementary metamaterial structures," Opt. Lett., vol. 39, no. 7, pp. 1709-1712, 2014.

[19] Y.-J. Chiang, C.-S. Yang, Y.-H. Yang, C.-L. Pan, and T.-J. Yen, “An ultrabroad terahertz bandpass filter based on multiple-resonance excitation of a composite metamaterial," Appl. Phys. Lett., vol. 99, no. 19, 2011.

[20] M. Lu, W. Li, and E. R. Brown, "Second-order bandpass terahertz filter achieved by multilayer complementary metamaterial structures," Opt. Lett., vol. 36, no. 7, pp. 1071-1073, 2011.

[21] O. Paul, R. Beigang, and M. Rahm, "Highly selective terahertz bandpass filters based on trapped mode excitation," Opt. Express, vol. 17, no. 21, pp. 18 590-18 595, 2009.

[22] F. Bayatpur, "Metamaterial-inspired frequency-selective surfaces," $\mathrm{Ph}$ D. dissertation, The University of Michigan, Ann Arbor, MI, USA, 2009.

[23] K. Sarabandi and N. Behdad, "A frequency selective surface with miniaturized elements," IEEE Trans. Antennas Propagat., vol. 55, no. 5, pp. 1239-1245, May 2007.

[24] F. Bayatpur and K. Sarabandi, "Tuning performance of metamaterialbased frequency selective surfaces," IEEE Trans. AntennasPropagat., vol. 57, no. 2, pp. 590-592, Feb. 2009.

[25] A. Ebrahimi, W. Withayachumnankul, S. Al-Sarawi, and D. Abbott, "Design of dual-band frequency selective surface with miniaturized elements," in IEEE Int. Workshop on Antenna Technol. (iWAT), 2014, pp. 206-209.

[26] F. Bayatpur and K. Sarabandi, "Multipole spatial filters using metamaterial-based miniaturized-element frequency-selective surfaces," IEEE Trans. Microw. Theory Techn., vol. 56, no. 12, pp. 2742-2747, Dec. 2008.

[27] C.-N. Chiu and K.-P. Chang, "A novel miniaturized-element frequency selective surface having a stable resonance," IEEE Antennas Wireless Propagat. Lett., vol. 8, pp. 1175-1177, 2009.

[28] A. Ebrahimi, P. Yaghmaee, W. Withayachumnankul, C. Fumeaux, S. Al-Sarawi, and D. Abbott, "Interlayer tuning of X-band frequencyselective surface using liquid crystal," in Proc. Asia-Pacific Microw. Conf. (APMC), 2013, pp. 1118-1120.

[29] M. Kashanianfard and K. Sarabandi, "Metamaterial inspired optically transparent band-selective ground planes for antenna applications," IEEE Trans. Antennas Propagat., vol. 61, no. 9, pp. 4624-4631, Sep. 2013.

[30] B. Li and Z. Shen, "Synthesis of quasi-elliptic bandpass frequencyselective surface using cascaded loop arrays," IEEE Trans. Antennas Propagat., vol. 61, no. 6, pp. 3053-3059, 2013.

[31] M. Li and N. Behdad, "Frequency selective surfaces for pulsed highpower microwave applications," IEEE Trans. Antennas Propagat., vol. 61, no. 2, pp. 677-687, 2013.

[32] S. Momeni Hasan Abadi, M. Li, and N. Behdad, "Harmonic-suppressed miniaturized-element frequency selective surfaces with higher order bandpass responses," IEEE Trans. Antennas Propagat., vol. 62, no. 5, pp. 2562-2571, May 2014. 
[33] M. Salehi and N. Behdad, "A second-order dual X-/Ka-band frequency selective surface,” IEEE Microw. Wireless Components Lett., vol. 18, no. 12 , pp. $785-787$, Dec. 2008.

[34] M. Moallem and K. Sarabandi, "Miniaturized-element frequency selective surfaces for millimeter-wave to terahertz applications," IEEE Trans. THz Sci. Technol., vol. 2, no. 3, pp. 333-339, May 2012.

[35] A. Bromel, U. Schinkel, S. Anders, T. May, and H. Meyer, "Polymer filters for far-infrared spectroscopy," in 37th Int. Conf. on Infrared, Millim. THz Waves (IRMMW-THz), Sep. 2012.

[36] M. A. Al-Joumayly and N. Behdad, "A generalized method for synthesizing low-profile, band-pass frequency selective surfaces with nonresonant constituting elements," IEEE Trans. Antennas Propagat., vol. 58, no. 12, pp. 4033-4041, Dec. 2010.

[37] N. Behdad and M. A. Al-Joumayly, "A generalized synthesis procedure for low-profile, frequency selective surfaces with odd-order bandpass responses," IEEE Trans. Antennas Propagat., vol. 58, no. 7, pp. 2460-2464, 2010.

[38] M. Al-Joumayly and N. Behdad, "A new technique for design of lowprofile, second-order, bandpass frequency selective surfaces," IEEE Trans. Antennas Propagat., vol. 57, no. 2, pp. 452-459, 2009.

[39] D. M. Pozar, Microwave Engineering. Hoboken, NJ, USA: Wiley, 2009.

[40] A. I. Zverev, Handbook of Filter Synthesis. New York, NY, USA: Wiley, 1967, vol. 47.

[41] D. Cavallo, W. Syed, and A. Neto, "Closed-form analysis of artificial dielectric layers-Part I: Properties of a single layer under planewave incidence," IEEE Trans. Antennas Propagat., vol. 62, no. 12, pp. 6256-6264, Dec. 2014.

[42] D. Cavallo, W. Syed, and A. Neto, "Closed-form analysis of artificial dielectric layers-Part II: Extension to multiple layers and arbitrary illumination," IEEE Trans. Antennas Propagat., vol. 62, no. 12, pp. 6265-6273, Dec. 2014

[43] N. Behdad, "A second-order band-pass frequency selective surface using nonresonant subwavelength periodic structures," Microw. Optical Technol. Lett., vol. 50, no. 6, pp. 1639-1643, 2008.

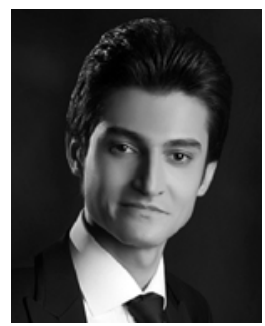

Amir Ebrahimi (S'10-GSM'10-M'11) ) was born in Babol, Iran, on September 11, 1986. He received the B.Sc. degree in electrical and computer engineering from the University of Mazandaran, Iran, in 2008, and the M.Sc. degree in electronic engineering from Babol University of Technology, Babol, Iran, 2011, and is currently working toward the Ph.D. degree in electrical and electronic engineering at the University of Adelaide, South Australia.

From 2009 to 2012, he was a research assistant in Integrated Circuits Research Laboratory (ICRL), Babol University of Technology. During 2014-2015, he was a visiting research fellow at Nanyang Technological University (NTU), Singapore. His research interests include metamaterial-inspired microwave devices, microwave circuit design, microwave filters, frequency selective surfaces (FSSs) and nonlinear RF and microwave circuits design and analysis.

Mr. Ebrahimi is a recipient of an International Postgraduate Research (Ph.D.) Scholarship (IPRS) by the Australian Government (2012), Australian Postgraduate Award (APA) (2012), Australian National Fabrication Facility (ANFF) award for fabricating high performance microwave microfluidic sensors in collaboration with the Ian Wark Research Institute, University of South Australia (2013), and the University of Adelaide D. R. Stranks Traveling Fellowship (2014).

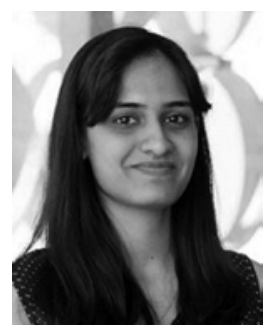

Shruti Nirantar (S'09) received the M.S degree in electrical and electronics engineering from the Royal Ielbourne Intitute of Technology (RMIT) University, Melbourne, Australia, in 2014. Her research interest is in Terahertz Micro-devices.

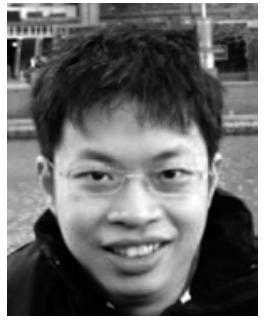

Withawat Withayachumnankul (GSM'08-M'09) received the B.Eng. and M.Eng degrees in electronic engineering from King Mongkut's Institute of Technology Ladkrabang (KMITL), Bangkok, Thailand, in 2001 and 2003, respectively, and the Ph.D. degree in electrical engineering (with commendation) from the University of Adelaide, Adelaide, Australia, in 2010.

From 2003 to 2012, he served as a Lecturer at KMITL with the Faculty of Engineering. From 2010-2013, he has held an ARC Australian Postdoctoral Fellowship with the University of Adelaide. Since 2014, he has become a lecturer at the University of Adelaide. Currently, he is also a JSPS Visiting Fellow at Tokyo Institute of Technology, Japan, and an Associate of RMIT University, Melbourne, Australia. His research interests include terahertz technology, metamaterials, plasmonics, and optical antennas. He has authored and co-authored more than 40 journal publications. He has delivered invited talks at ETH Zürich (ETHZ), University of Marburg, Universität Kaiserslautern, EPFL, IPHT (Germany), The University of Western Australia, and Ibaraki University.

Dr. Withayachumnankul serves as a grant assessor for Swiss National Science Foundation (SNSF), German Academic Exchange Service (DAAD), and Australian Research Council (ARC). He is a recipient of the IEEE/LEOS Graduate Student Fellowship (2008) and the SPIE Scholarship in Optical Science and Engineering (2008).

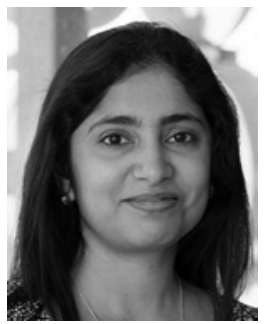

Madhu Bhaskaran (S'04-GSM'05-M'09) received the Ph.D. degree from the Functional Materials and Microsystems Research Group at RMIT University, Melbourne, Australia, in 2009.

She jointly leads the Functional Materials and Microsystems Research Group at RMIT University, Australia. Her research interests include two-dimensional materials, flexible electronics, functional oxides, and micro/nano-electronics. She has published 70 peer-reviewed journal articles.

Dr. Bhaskaran is the recipient of a 2010-2014 Australian Post-Doctoral Fellowship from the Australian Research Council, the 2011 RMIT Vice-Chancellor's Early Career Researcher Award, the 2011 RMIT Media Star Award for Research, and the 2014 Phillip Law Post-Doctoral Award for Physical Sciences awarded by the Royal Society of Victoria.

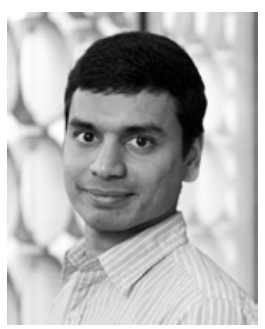

Sarath Sriram (S'04-GSM'05-M'09) received the Ph.D. degree from the Functional Materials and Microsystems Research Group at RMIT University, Melbourne, Australia, in 2009

$\mathrm{He}$ is the joint leader of the Functional Materials and Microsystems Research Group at RMIT University, Melbourne, Australia. He is also the Deputy Director of the University's Micro Nano Research Facility, a 1,200 sq.m. state-of-the-art micro/nanofabrication capability. His expertise includes the synthesis of functional thin films and micro/nanostructures and devices. He has published more than 100 peer-reviewed publications.

Dr. Sriram is the recipient of the 2010 Gold Medal for Excellence in Research from the Australian Institute of Nuclear Science and Engineering, a 2011-2014 Australian Post-Doctoral Fellowship from the Australian Research Council, the 2012 NMI Prize for Measurement Excellence from the National Measurement Institute, Australia, a 2012 Victoria Fellowship, and the 2014 RMIT Vice-Chancellor's Early Career Researcher Award. 


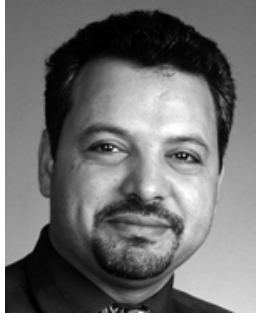

Said F. Al-Sarawi (S'92-M'01) received the general certificate in marine radio communication and the B.Eng. degree (1st Class Hons.) in marine electronics and communication from the Arab Academy for Science and Technology (AAST), Egypt, in 1987 and 1990, respectively, and the Ph.D. degree in mixed analog and digital circuit design techniques for smart wireless systems with special commendation in electrical and electronic engineering, in 2003, from The University of Adelaide, Australia.

Currently, he is the Director of the Centre for Biomedical Engineering and a founding member of Education Research Group of Adelaide (ERGA) in the University of Adelaide. His research interests include design techniques for mixed signal systems in CMOS and optoelectronic technologies for high performance radio transceivers, low power and low voltage radio frequency identification (RFID) systems, data converters, mixed signal design, and microelectromechanical systems (MEMS) for biomedical applications. His current educational research is focused on innovative teaching techniques for engineering education, research skill development, and factors affecting students evaluations of courses in different disciplines.

Dr. Al-Sarawi was awarded The University of Adelaide Alumni Postgraduate Medal (formerly Culross Prize) for outstanding academic merit at the postgraduate level. While pursuing his Ph.D., he won the Commonwealth Postgraduate Research Award (Industry). He also received the Graduate Certificate in Education (Higher Education), in 2006, from the same university.

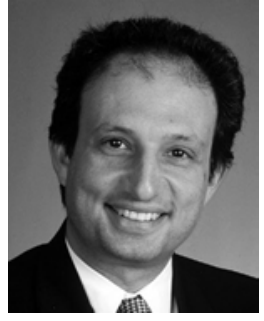

Derek Abbott (M'85-SM'99-F'05) was born in South Kensington, London, U.K., in 1960. He received the B.Sc. (honors) degree in physics fromLoughborough University, Loughborough, Leicestershire, U.K., in 1982 and the Ph.D. degree in electrical and electronic engineering from The University of Adelaide, Adelaide, S.A. Australia, in 1995, under K. Eshraghian and B. R. Davis.

From 1978 to 1986, he was a Research Engineer at the GEC Hirst Research Centre, London, U.K. From 1986 to 1987 , he was a VLSI Design Engineer at Austek Microsystems, Australia. Since 1987, he has been with The University of Adelaide, where he is presently a full Professor with the School of Electrical and Electronic Engineering. He coedited Quantum Aspects of Life (Imperial College Press, 2008), coauthored Stochastic Resonance (Cambridge University Press, 2012), and coauthored Terahertz Imaging for Biomedical Applications (Springer-Verlag, 2012). He holds over 800 publications/patents and has been an invited speaker at over 100 institutions. Prof. Abbott's interests are in the area of multidisciplinary physics and electronic engineering applied to complex systems. His research programs span a number of areas of stochastics, game theory, photonics, biomedical engineering, and computational neuroscience.

Prof Abbott is a Fellow of the Institute of Physics (IOP). He has won a number of awards including the South Australian Tall Poppy Award for Science (2004), the Premier's SA Great Award in Science and Technology for outstanding contributions to South Australia (2004), and an Australian Research Council (ARC) Future Fellowship (2012). He has served as an Editor and/or Guest Editor for a number of journals including IEEE JOURNAL OF SOLID-STATE Circuits, Journal of Optics B, Microelectronics Journal, Chaos, Smart Structures and Materials, Fluctuation Noise Letters, PLOS ONE, and is currently on the editorial boards of IEEE ACCESS, Nature, Scientific Reports, and Royal Society Open Science. 